\title{
Optimal Resource Allocation in Full-Duplex Ambient Backscatter Communication Networks for Green IoT
}

\author{
Gang Yang, Member, IEEE, Dongdong Yuan, and Ying-Chang Liang, Fellow, IEEE \\ University of Electronic Science and Technology of China, Chengdu, P. R. China \\ Email: yanggang@uestc.edu.cn, 201721260420@std.uestc.edu.cn, liangyc@ieee.org
}

\begin{abstract}
Ambient backscatter communication (AmBC) enables wireless-powered backscatter devices (BDs) to transmit information over ambient radio-frequency (RF) carriers without using an RF transmitter, and thus has emerged as a promising technology for green Internet-of-Things. This paper considers an AmBC network in which a full-duplex access point (FAP) simultaneously transmits downlink orthogonal frequency division multiplexing (OFDM) signals to its legacy user (LU) and receives uplink signals backscattered from multiple BDs in a time-division-multiple-access manner. To enhance the system performance from multiple design dimensions and ensure fairness, we maximize the minimum throughput among all BDs by jointly optimizing the BDs' backscatter time portions, the BDs' power reflection coefficients, and the FAP's subcarrier power allocation, subject to the LU's throughput constraint, the BDs' harvested-energy constraints, and other practical constraints. As such, we propose an efficient iterative algorithm for solving the formulated non-convex problem by leveraging the block coordinated decent and successive convex optimization techniques. We further show the convergence of the proposed algorithm, and analyze its complexity. Finally, extensive simulation results show that the proposed joint design achieves significant throughput gains as compared to the benchmark scheme with equal resource allocation.
\end{abstract}

\section{INTRODUCTION}

Internet of Things (IoT) is a key application scenario for the fifth generation $(5 \mathrm{G})$ and future mobile communication systems, and various IoT devices typically have strict limitations on energy, cost, and complexity. Recently, ambient backscatter communication (AmBC) which enables backscatter devices (BDs) to modulate their information symbols over the ambient RF carriers (e.g., WiFi, TV, or cellular signals) without using a complex and powerhungry RF transmitter [1], has emerged as a promising technology for energy-efficient and cost-efficient IoT communications.

The existing AmBC systems can be divided into three categories, the standard AmBC system with separated backscatter receiver and ambient transmitter (its legacy receiver) [2]-[6], the cooperative AmBC system with co-located backscatter receiver and legacy receiver [7], [8], and the full-duplex AmBC system with co-located backscatter receiver and ambient transmitter [9]. Existing works on AmBC focus on the transceiver design and hardware prototype. To address the problem of strong direct-link interference from ambient transmitter in standard AmBC systems, some studies shift the backscattered signal to a clean band that does not overlap with the direct-link signal [5], [6]. In [3], the BD waveform and backscatter receiver detector are jointly designed to cancel out the direct-link interference from the ambient orthogonal frequency division multiplexing (OFDM) signals. A full-duplex AmBC system is proposed in [9], in which the WiFi access point decodes the received backscattered signal while simultaneously transmitting WiFi packages to its legacy client.

Recently, there are a few literature on performance analysis and optimization for AmBC systems. In [10], the ergodic capacity for backscatter communication is maximized for a standard AmBC system. In [8], the transmit beamforming is optimized for a cooperative AmBC system with multiple antennas at the ambient transmitter. In [11], for an ambient-backscatter assisted cognitive radio network, the secondary transmitter's rate is maximized by optimizing the time resource.

In this paper, we consider a full-duplex AmBC network $(\mathrm{ABCN})$ over ambient OFDM carriers as shown in Fig. 1 , consisting of a full-duplex access point (FAP), a legacy user (LU), and multiple BDs. We optimize the throughput performance for such an $\mathrm{ABCN}$, which has not been studied in the literature to our best knowledge. The main contributions of this paper are summarized as follows:

- First, by employing an FAP, we propose a new model to enable simultaneous downlink information transmission (energy transfer) to the LU (multiple BDs) and uplink information transmission from multiple BDs. We characterize the corresponding throughput and energy transfer performances of the BDs, as well as the throughput performance of the LU.

- Second, to ensure fairness, we formulate a problem to maximize the minimum throughput among all BDs by jointly optimizing three blocks of variables including the BDs' backscatter time portions, the BDs' power 
reflection coefficients, and the FAP's subcarrier power allocation. Through joint optimization, the system performance can benefit from multiple design dimensions.

- Third, to solve the formulated non-convex problem, we propose an iterative algorithm by leveraging the block coordinated decent (BCD) and successive convex optimization (SCO) techniques, in each iteration of which the three blocks of variables are alternately optimized. Also, we show the convergence of the proposed algorithm and analyze its complexity.

- Finally, numerical results show that significant throughput gains are achieved by our proposed joint design, as compared to the benchmark scheme with equal resource allocation. Also, the BD-LU throughput tradeoff and the BDs' throughput-energy tradeoff are revealed.

The rest of this paper is organized as follows. Section II presents the system model for a full-duplex ABCN over ambient OFDM carriers. Section III formulates the minimum-throughput maximization problem. Section IV proposes an efficient iterative algorithm by applying the BCD and SCO techniques. Section $\nabla$ presents the numerical results. Finally, Section VI concludes this paper.

\section{SYSTEM MODEL}

In this section, we present the system model for a full-duplex ABCN. As illustrated in Fig. 1, we consider two co-existing communication systems: the legacy communication system which consists of an FAP with two antennas for simultaneous information transmission and reception, respectively, and its dedicated $\mathrm{LU}^{\mathrm{I}}$, and the AmBC system which consists of the FAP and $M$ BDs. The FAP transmits OFDM signals to the LU. We are interested in the AmBC system in which each BD transmits its modulated signal back to the FAP over its received ambient OFDM carrier. Each BD contains a backscatter antenna, a switched load impedance, a micro-controller, a signal processor, an energy harvester, and other modules (e.g., battery, memory, sensing). The BD modulates its received ambient OFDM carrier by intentionally switching the load impedance to vary the amplitude and/or phase of its backscattered signal, and the backscattered signal is received and finally decoded by the FAP. The energy harvester collects energy from ambient OFDM signals and uses it to replenish the battery which provides power for all modules of the BD.

The block fading channel model is assumed. As shown in Fig. 1, let $f_{m, l}$ be the $L_{\mathrm{f}}$-path forward channel response from the FAP to the $m$-th $\mathrm{BD}$, for $m=1, \ldots, M, g_{m, l}$ be the $L_{\mathrm{g}}$-path backward channel response from the $m$-th $\mathrm{BD}$

\footnotetext{
${ }^{1}$ We consider the case of a single LU, since the FAP typically transmits to an LU in a short period for practical OFDM systems like WiFi system. The analyses and results can be extended to the multiple-LU case.
}

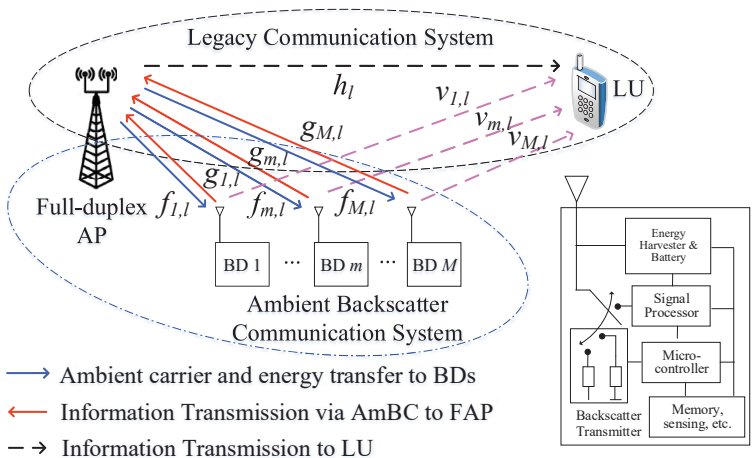

$\rightarrow$ Information Transmission to LU

$\rightarrow$ Interference to LU

Backscatter Device

Fig. 1: System description for a full-duplex ABCN.

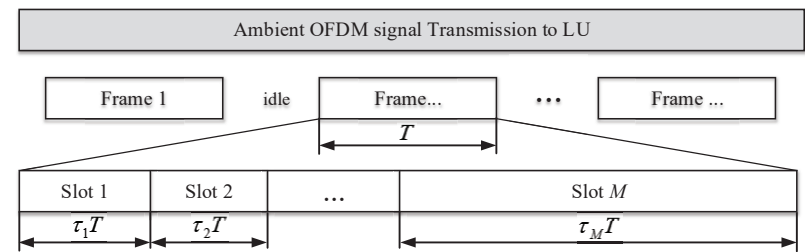

Fig. 2: Frame structure for TDMA-based ABCN.

to the FAP, $h_{l}$ be the $L_{\mathrm{h}}$-path direct-link channel response from the FAP to the $\mathrm{LU}$, and $v_{m, l}$ be the $L_{\mathrm{v}}$-path interference channel response from the $m$-BD to the LU. Let $N$ be the number of subcarriers of the transmitted OFDM signals. For each channel, define the frequency response at the $k$-th subcarrier as $F_{m, k}=\sum_{l=0}^{L_{\mathrm{f}}-1} f_{m, l} e^{\frac{-j 2 \pi k l}{N}}$, $G_{m, k}=\sum_{l=0}^{L_{\mathrm{g}}-1} g_{m, l} e^{\frac{-j 2 \pi k l}{N}}, V_{m, k}=\sum_{l=0}^{L_{\mathrm{v}}-1} v_{m, l} e^{\frac{-j 2 \pi k l}{N}}$, $H_{k}=\sum_{l=0}^{L_{h}-1} h_{l} e^{\frac{-j 2 \pi k l}{N}}$, for $k=0, \ldots, N-1$.

We consider frame-based transmission, and the frame structure is shown in Fig. 2, In each frame of time duration $T$ (seconds) consisting of $M$ slots, the FAP simultaneously transmits downlink OFDM signals to the LU, and receives uplink signals backscattered from all BDs in a timedivision-multiple-access (TDMA) manner. The $m$-th slot of time duration $\tau_{m} T$ (with time portion $\tau_{m}\left(0 \leq \tau_{m} \leq 1\right)$ ) is assigned to the $m$-th BD. Denote the backscatter time portion vector $\boldsymbol{\tau}=\left[\begin{array}{llll}\tau_{1} & \tau_{2} & \ldots & \tau_{M}\end{array}\right]^{T}$. In the $m$-th slot, BD $m$ reflects back a portion of its incident signal for transmitting information to the FAP and harvests energy from the remaining incident signal, and all other BDs only harvest energy from their received OFDM signals.

Let $S_{m, k}(n) \in \mathcal{C}$ be the FAP's information symbol at the $k$-th subcarrier, $\forall k$, in the $n$-th OFDM symbol period of the $m$-th slot. After inverse discrete Fourier transform at the FAP, a cyclic-prefix (CP) of length $N_{\mathrm{cp}}$ is added at the beginning of each OFDM symbol. The transmitted time-domain signal in each OFDM symbol period is

$$
s_{m, t}(n)=\frac{1}{N} \sum_{k=0}^{N-1} \sqrt{P_{m, k}} S_{m, k}(n) e^{j 2 \pi \frac{k t}{N}},
$$

for the time index $t=0,1, \ldots, N-1$, where $P_{m, k}$ is the allocated power at the $k$-th subcarrier in the $m$-th slot. The 
subcarrier power values are subject to the average power constraint $\sum_{m=1}^{M} \tau_{m} \sum_{k=0}^{N-1} P_{m, k} \leq \bar{P}$, where $\bar{P}$ is the total transmission power in all slots. Denote the subcarrier power allocation matrix $\mathbf{P}=\left[\begin{array}{llll}\mathbf{p}_{1} & \mathbf{p}_{2} & \ldots & \mathbf{p}_{M}\end{array}\right]$, where $\mathbf{p}_{m}$ is the subcarrier power allocation vector in the $m$-th slot.

In the $m$-th slot, the incident signal at $\mathrm{BD} m$ is $s_{m, t}(n) \otimes f_{m, l}$, where $\otimes$ means the convolution operation. Let $\alpha_{m}\left(0 \leq \alpha_{m} \leq 1\right)$ be the $m$-th BD's power reflection coefficient, and denote the vector $\boldsymbol{\alpha}=\left[\begin{array}{llll}\alpha_{1} & \alpha_{2} \ldots \alpha_{M}\end{array}\right]^{T}$. Let $\eta(0 \leq \eta \leq 1)$ be the energy-harvesting efficiency for all BDs. From the aforementioned system model and [12], the total harvested energy by BD $m$ in all slots is thus

$$
\begin{aligned}
& E_{m}\left(\boldsymbol{\tau}, \alpha_{m}, \mathbf{P}\right)= \\
& \eta \sum_{k=0}^{N-1}\left|F_{m, k}\right|^{2}\left[\tau_{m} P_{m, k}\left(1-\alpha_{m}\right)+\sum_{r=1, \neq m}^{M} \tau_{r} P_{r, k}\right] .
\end{aligned}
$$

Let $X_{m}(n)$ be the $m$-th BD's information symbol, whose duration is designed to be the same as the OFDM symbol period. We assume each BD can align the transmission of its own symbol $X_{m}(n)$ with its received OFDM symbo 2 . In the $m$-th slot, the backscattered signal from the $m$-th BD is thus $\sqrt{P_{m, k}} \sqrt{\alpha_{m}} s_{m, t}(n) \otimes f_{m, l} X_{m}(n)$.

The transmitted downlink signal $s_{m, t}$ is known by the FAP's receiving chain. Thus, this signal can be reconstructed and subtracted from the received signals. Therefore, the self-interference can be cancelled by using existing digital or analog cancellation techniques. For this reason, we assume perfect self-interference cancellation (SIC) at the FAP in this paper. After performing SIC, the received signal backscattered from the $m$-th $\mathrm{BD}$ is given by

$$
\begin{aligned}
& y_{m, t}(n)= \\
& \quad \sqrt{P_{m, k}} \sqrt{\alpha_{m}} s_{m, t}(n) \otimes f_{m, l} \otimes g_{m, l} X_{m}(n)+w_{m, t}(n),
\end{aligned}
$$

where the additive white Gaussian noise (AWGN) is assumed, i.e., $w_{m, t}(n) \sim \mathcal{C} \mathcal{N}\left(0, \sigma^{2}\right)$.

After CP removal and discrete Fourier transform operation at the FAP, the received frequency-domain signal is

$$
\begin{aligned}
& Y_{m, k}(n)= \\
& \quad \sqrt{P_{m, k}} \sqrt{\alpha_{m}} F_{m, k} G_{m, k} S_{m, k}(n) X_{m}(n)+W_{m, k}(n),
\end{aligned}
$$

where the frequency-domain noise $W_{m, k}(n) \sim \mathcal{C N}\left(0, \sigma^{2}\right)$.

The FAP performs maximum-ratio-combining (MRC) to recover the BD symbol $X_{m}(n)$ as follows

$$
\widehat{X}_{m}(n)=\frac{1}{N} \sum_{k=0}^{N-1} \frac{Y_{m, k}(n)}{\sqrt{P_{m, k}} \sqrt{\alpha_{m}} F_{m, k} G_{m, k} S_{m, k}},
$$

and the resulting decoding signal-to-noise-ratio (SNR) is

$$
\gamma_{m}\left(\alpha_{m}, \mathbf{P}\right)=\frac{\alpha_{m}}{\sigma^{2}} \sum_{k=0}^{N-1}\left|F_{m, k}\right|^{2}\left|G_{m, k}\right|^{2} P_{m, k} .
$$

\footnotetext{
${ }^{2} \mathrm{BD}$ can practically estimate the arrival time of OFDM signal by some
} methods like the scheme that utilizes the repeating structure of $\mathrm{CP}[3]$.
Hence, the $m$-th BD's throughpu目 normalized to $T$ is

$$
\begin{aligned}
& R_{m}\left(\tau_{m}, \alpha_{m}, \mathbf{p}_{m}\right)= \\
& \frac{\tau_{m}}{N} \log \left(1+\frac{\alpha_{m}}{\sigma^{2}} \sum_{k=0}^{N-1}\left|F_{m, k}\right|^{2}\left|G_{m, k}\right|^{2} P_{m, k}\right) .
\end{aligned}
$$

Similar to (4), the received frequency-domain signal at the LU can be written as follows

$Z_{m, k}(n)=\sqrt{P_{m, k}} H_{k} S_{m, k}(n)+\ldots$
$\sqrt{P_{m, k}} \sqrt{\alpha_{m}} F_{m, k} V_{m, k} S_{m, k}(n) X_{m}(n)+\widetilde{W}_{m, k}(n), \forall k, m$

where the frequency-domain noise $\widetilde{W}_{m, k}(n) \sim \mathcal{C N}\left(0, \sigma^{2}\right)$.

Similar to (7), treating backscatter-link signal as interference, the total throughput of the LU is given by

$$
\begin{aligned}
& \widetilde{R}(\boldsymbol{\tau}, \boldsymbol{\alpha}, \mathbf{P})= \\
& \frac{1}{N} \sum_{m=1}^{M} \tau_{m} \sum_{k=0}^{N-1} \log \left(1+\frac{\left|H_{k}\right|^{2} P_{m, k}}{\alpha_{m}\left|F_{m, k} V_{m, k}\right|^{2} P_{m, k}+\sigma^{2}}\right) .
\end{aligned}
$$

\section{PROBLEM Formulation}

In this section, we formulate the optimization problem. The objective is to maximize the minimum throughput $Q(\boldsymbol{\tau}, \boldsymbol{\alpha}, \mathbf{P}) \triangleq \min _{m=1, \ldots, M} R_{m}\left(\tau_{m}, \alpha_{m}, \mathbf{p}_{m}\right)$ among all BDs, by jointly optimizing the BDs' backscatter time portions (i.e., $\boldsymbol{\tau}$ ), the BD's power reflection coefficients (i.e., $\boldsymbol{\alpha}$ ), and the FAP's subcarrier power allocation (i.e., $\mathbf{P}$ ). We consider the following constraints: the total throughput of the LU needs to be larger than a given minimum throughput $D$, i.e., $\widetilde{R}(\boldsymbol{\tau}, \boldsymbol{\alpha}, \mathbf{P}) \geq D$; each $\mathrm{BD}$ has a minimum energy requirement $E_{\min , m}$, i.e., $E_{m}\left(\boldsymbol{\tau}, \alpha_{m}, \mathbf{P}\right) \geq$ $E_{\text {min }, m}, \forall m$; the total power consumed by the FAP needs to be less than a given maximum power $\bar{P}$, i.e., $\sum_{m=1}^{M} \sum_{k=0}^{N-1} \tau_{m} P_{m, k} \leq \bar{P}$; the sum of backscatter time portions for all BDs should be no larger than 1, i.e., $\sum_{m=1}^{M} \tau_{m} \leq 1$, with non-negative time portion $\tau_{m}$ for each BD $m$; the peak power value for each subcarrier is $P_{\text {peak }}$, i.e., $0 \leq P_{m, k} \leq P_{\text {peak }}, \forall m, k$; the power reflection coefficients are positive numbers and no larger than 1, i.e., $0 \leq \alpha_{m} \leq 1, \forall m$. The optimization problem is thus formulated as follows

$$
\begin{aligned}
& \max _{Q, \boldsymbol{\tau}, \boldsymbol{\alpha}, \mathbf{P}} Q \\
& \text { s.t. } \quad \frac{\tau_{m}}{N} \log \left(1+\frac{\alpha_{m}}{\sigma^{2}} \sum_{k=0}^{N-1}\left|F_{m, k}\right|^{2}\left|G_{m, k}\right|^{2} P_{m, k}\right) \geq Q, \forall m
\end{aligned}
$$

$$
\frac{1}{N} \sum_{m=1}^{M} \tau_{m} \sum_{k=0}^{N-1} \log \left(1+\frac{\left|H_{k}\right|^{2} P_{m, k}}{\alpha_{m}\left|F_{m, k} V_{m, k}\right|^{2} P_{m, k}+\sigma^{2}}\right) \geq D
$$

\footnotetext{
${ }^{3}$ This paper adopts normalized throughput with unit of $\mathrm{bps} / \mathrm{Hz}$.
} 


$$
\begin{aligned}
& \eta \sum_{k=0}^{N-1}\left|F_{m, k}\right|^{2}\left[\tau_{m} P_{m, k}\left(1-\alpha_{m}\right)+\sum_{r=1, \neq m}^{M} \tau_{r} P_{r, k}\right] \\
& \quad \geq E_{\text {min }, m}, \forall m \\
& \sum_{m=1}^{M} \sum_{k=0}^{N-1} \tau_{m} P_{m, k} \leq \bar{P} \\
& \sum_{m=1}^{M} \tau_{m} \leq 1 \\
& 0 \leq P_{m, k} \leq P_{\text {peak }}, \quad \forall m, k \\
& \tau_{m} \geq 0, \quad \forall m \\
& 0 \leq \alpha_{m} \leq 1, \quad \forall m .
\end{aligned}
$$

Notice that problem (10) is non-convex and challenging to solve in general, since the variables are all coupled and the constraint function in $10 \mathrm{c}$ is non-convex over $P_{m, k}$ 's.

\section{Proposed Algorithm}

In this section, we propose an efficient iterative algorithm for the problem (10) by applying the block coordinate descent (BCD) [13] and successive convex optimization (SCO) techniques [14]. Then, we show the convergence of the proposed algorithm and analyze its complexity.

\section{A. Backscatter Time Allocation Optimization}

In iteration $j$, for given power reflection coefficients $\boldsymbol{\alpha}^{\{j\}}$ and subcarrier power allocation $\mathbf{P}^{\{j\}}$, the backscatter time portions $\tau$ can be optimized by solving the problem

$$
\begin{aligned}
& \max _{Q, \tau} Q \\
& \text { s.t. } 10 \mathrm{~b}, 10 \mathrm{c}, 10 \mathrm{~d}, 10 \mathrm{~d}, 10 \mathrm{f}, 10 \mathrm{~h},
\end{aligned}
$$

where the variables $P_{m, k}$ 's and $\alpha_{m}$ 's are replaced by given $P_{m, k}^{\{j\}}$,s and $\alpha_{m}^{\{j\}}$,s, respectively, in all the constraints. Notice that problem (11) is a standard linear programming (LP), it can be solved efficiently by existing optimization tools such as CVX [15].

\section{B. Reflection Power Allocation Optimization}

For given backscatter time portions $\boldsymbol{\tau}^{\{j\}}$ and subcarrier power allocation $\mathbf{P}^{\{j\}}$, the power reflection coefficients $\boldsymbol{\alpha}$ can be optimized by solving the following problem

$$
\begin{aligned}
& \max _{Q, \alpha} Q \\
& \text { s.t. 10b, 10c, 10d, 10i), }
\end{aligned}
$$

where the variables $P_{m, k}$ 's and $\tau_{m}$ 's are replaced by given $P_{m, k}^{\{j\}}$,s and $\tau_{m}^{\{j\}}$,s, respectively. Since the left-hand-side of the constraint (10c) with given $P_{m, k}^{\{j\}}$ and $\tau_{m}^{\{j\}}$ is a decreasing and convex function of $\alpha_{m}$, the constraint is convex. Hence, problem (12) is a convex optimization problem that can also be efficiently solved by CVX [15].

\section{Subcarrier Power Allocation Optimization}

For given backscatter time portions $\boldsymbol{\tau}^{\{j\}}$ and power reflection coefficients $\boldsymbol{\alpha}^{j}$, the subcarrier power allocation $\mathbf{P}$ can be optimized by solving the following problem

$$
\begin{aligned}
& \max _{Q, \mathbf{P}} \quad Q \\
& \text { s.t. } \quad \frac{1}{N} \sum_{m=1}^{M} \tau_{m}^{\{j\}} \\
& \quad \sum_{k=0}^{N-1} \log \left(1+\frac{\left|H_{k}\right|^{2} P_{m, k}}{\alpha_{m}^{\{j\}}\left|F_{m, k} V_{m, k}\right|^{2} P_{m, k}+\sigma^{2}}\right) \geq D \\
& \quad(10 \mathrm{~b},(10 \mathrm{~d}),(10 \mathrm{e}),(10 \mathrm{~g}),
\end{aligned}
$$

where the variables $\tau_{m}$ 's and $\alpha_{m}$ 's are replaced by given values $\tau_{m}^{\{j\}}$,s and $\alpha_{m}^{\{j\}}$,s, respectively. Since the constraint function $\left.\widetilde{R}(\mathbf{P})\right|_{\boldsymbol{\tau}\{j\}, \boldsymbol{\alpha}\{j\}}$ in (13b) is non-convex with respect to $P_{m, k}$, problem (13) is non-convex. Notice that the constraint function $\left.\widetilde{R}(\mathbf{P})\right|_{\boldsymbol{\tau}^{\{j\}}, \boldsymbol{\alpha}^{\{j\}}}$ can be rewritten as

$$
\begin{aligned}
& \left.\widetilde{R}(\mathbf{P})\right|_{\boldsymbol{\tau}^{\{j\}}, \boldsymbol{\alpha}\{j\}} \\
& =\sum_{m=1}^{M} \frac{\tau_{m}^{\{j\}}}{N} \sum_{k=0}^{N-1}\left[-\log \left(\alpha_{m}\left|F_{m, k} V_{m, k}\right|^{2} P_{m, k}+\sigma^{2}\right)+\ldots\right. \\
& \left.\quad \log \left(\left(\alpha_{m}^{\{j\}}\left|F_{m, k} V_{m, k}\right|^{2}+\left|H_{k}\right|^{2}\right) P_{m, k}+\sigma^{2}\right)\right] . \text { (14) }
\end{aligned}
$$

To handle the non-convex constraint (13b), we exploit the SCO technique [14] to approximate the second logarithm function in (14). Recall that any concave function can be globally upper-bounded by its first-order Taylor expansion at any point. Specifically, let $P_{m, k}^{\{j\}}$ denote the subcarrier power allocation in the last iteration. We have the following concave lower bound at the local point $P_{m, k}^{\{j\}}$

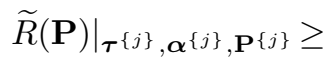

$\sum_{m=1}^{M} \frac{\tau_{m}^{\{j\}}}{N} \sum_{k=0}^{N-1}\left[\log \left(\left(\alpha\left|F_{m, k} V_{m, k}\right|^{2}+\left|H_{k}\right|^{2}\right) P_{m, k}+\sigma^{2}\right)-\ldots\right.$

$\log \left(\alpha\left|F_{m, k} V_{m, k}\right|^{2} P_{m, k}^{\{i, j\}}+\sigma^{2}\right)-$

$\left.\frac{\alpha\left|F_{m, k} V_{m, k}\right|^{2}\left(P_{m, k}-P_{m, k}^{\{i, j\}}\right)}{\alpha\left|F_{m, k} V_{m, k}\right|^{2} P_{m, k}^{\{i, j\}}+\sigma^{2}}\right]\left.\triangleq \widetilde{R}^{\mathrm{lb}}(\mathbf{P})\right|_{\boldsymbol{\tau}^{\{j\}}, \boldsymbol{\alpha}\{j\}, \mathbf{P}^{\{j\}}}$.

With given local points $\mathbf{P}^{\{j\}}$ and lower bound $\left.\widetilde{R}^{\mathrm{lb}}(\mathbf{P})\right|_{\boldsymbol{\tau}_{\{j\}}, \boldsymbol{\alpha}^{\{j\}}, \mathbf{P}\{j\}}$ in (15), by introducing the lowerbound minimum-throughput $Q_{\mathrm{tpa}}^{\mathrm{lb}}$, problem (13) is approximated as the following problem

$$
\begin{array}{ll}
\max _{Q_{\mathrm{tpa}}^{\mathrm{ba}}, \mathbf{P}} & Q_{\mathrm{tpa}}^{\mathrm{lb}} \\
\text { s.t. } & \left.\widetilde{R}^{\mathrm{lb}}(\mathbf{P})\right|_{\boldsymbol{\tau}^{\{j\}},}, \boldsymbol{\alpha}^{\{j\}}, \mathbf{P}^{\{j\}} \geq D \\
& 10 \mathrm{~b}, 10 \mathrm{~d}, 10 \mathrm{~d}, 10 \mathrm{~g},
\end{array}
$$

where the variables $\tau_{m}$ 's and $\alpha_{m}$ 's are replaced by given $\tau_{m}^{\{j\}}$, s and $\alpha_{m}^{\{j\}}$, s, respectively. Problem (16) is a convex 


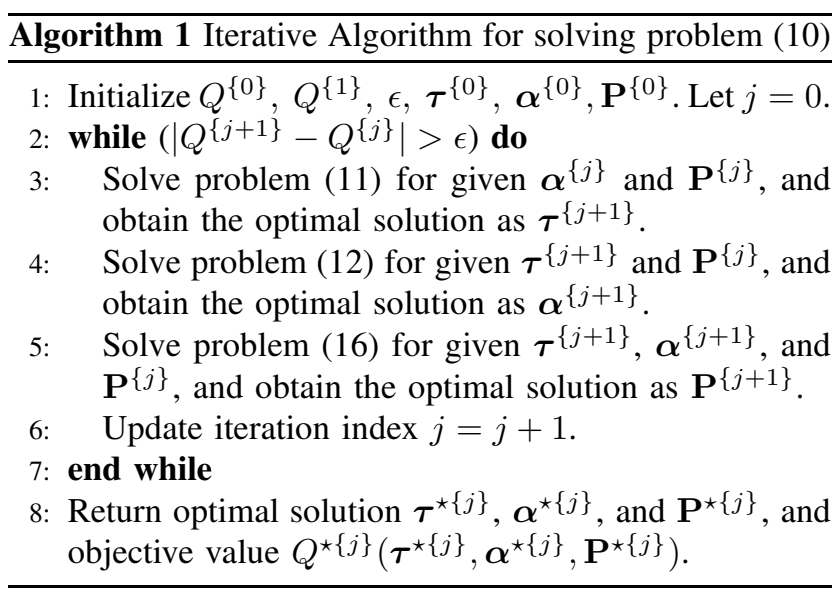

optimization problem which can also be efficiently solved by CVX [15]. It is noticed that the lower bound adopted in (16b) implies that the feasible set of problem (16) is always a subset of that of problem (13). As a result, the optimal objective value obtained from problem (16) is in general a lower bound of that of problem (13).

\section{Overall Algorithm}

We propose an overall iterative algorithm for problem (10) by applying the BCD technique [13]. Specifically, the entire variables in original problem (10) are partitioned into three blocks, i.e., $\boldsymbol{\tau}, \boldsymbol{\alpha}$, and $\mathbf{P}$, which are alternately optimized by solving problem (11), (12), and (16) correspondingly in each iteration, while keeping the other two blocks of variables fixed. Furthermore, the obtained solution in each iteration is used as the input of the next iteration. The details are summarized in Algorithm 1

\section{E. Convergence and Complexity Analysis}

Notice that in our case, for subcarrier power allocation problem (13), we only solve its approximate problem (16) optimally. Thus, the convergence analysis for the classical $\mathrm{BCD}$ technique cannot be directly applied [13], and the convergence of Algorithm 1 needs to be proved, as follows.

Theorem 1. Algorithm 1 is guaranteed to converge.

Proof. First, in step 3 of Algorithm 1, since the optimal solution $\boldsymbol{\tau}^{\{j+1\}}$ is obtained for given $\boldsymbol{\alpha}^{\{j\}}$ and $\mathbf{P}^{\{j\}}$, we have the following inequality on the minimum throughput $Q(\boldsymbol{\tau}, \boldsymbol{\alpha}, \mathbf{P})$

$$
Q\left(\boldsymbol{\tau}^{\{j\}}, \boldsymbol{\alpha}^{\{j\}}, \mathbf{P}^{\{j\}}\right) \leq Q\left(\boldsymbol{\tau}^{\{j+1\}}, \boldsymbol{\alpha}^{\{j\}}, \mathbf{P}^{\{j\}}\right) .
$$

Second, in step 4, since the optimal solution $\boldsymbol{\alpha}^{\{j+1\}}$ is obtained for given $\boldsymbol{\tau}^{\{j+1\}}$ and $\mathbf{P}^{\{j\}}$, it holds that

$$
Q\left(\boldsymbol{\tau}^{\{j+1\}}, \boldsymbol{\alpha}^{\{j\}}, \mathbf{P}^{\{j\}}\right) \leq Q\left(\boldsymbol{\tau}^{\{j+1\}}, \boldsymbol{\alpha}^{\{j+1\}}, \mathbf{P}^{\{j\}}\right) .
$$

Third, in step 5, it follows that

$$
Q\left(\boldsymbol{\tau}^{\{j+1\}}, \boldsymbol{\alpha}^{\{j+1\}}, \mathbf{P}^{\{j\}}\right) \stackrel{(a)}{=} Q_{\mathrm{tpa}}^{\mathrm{lb},\{j\}}\left(\boldsymbol{\tau}^{\{j+1\}}, \boldsymbol{\alpha}^{\{j+1\}}, \mathbf{P}^{\{j\}}\right)
$$

$$
\begin{aligned}
& \stackrel{(b)}{\leq} Q_{\mathrm{tpa}}^{\mathrm{lb},\{j\}}\left(\boldsymbol{\tau}^{\{j+1\}}, \boldsymbol{\alpha}^{\{j+1\}}, \mathbf{P}^{\{j+1\}}\right) \\
& \stackrel{(c)}{\leq} Q\left(\boldsymbol{\tau}^{\{j+1\}}, \boldsymbol{\alpha}^{\{j+1\}}, \mathbf{P}^{\{j+1\}}\right),(19)
\end{aligned}
$$

where (a) holds since the Taylor expansion in (15) is tight at given local point, which implies problem (16) at $\mathbf{P}^{\{j\}}$ has the same objective function as that of problem (13); (b) is because $\mathbf{P}^{\{j+1\}}$ is the optimal solution to problem (16); (c) holds since the objective value of problem (16) is a lower bound of that of its original problem (13).

From (17), 18), and (19), we have

$$
Q\left(\boldsymbol{\tau}^{\{j\}}, \boldsymbol{\alpha}^{\{j\}}, \mathbf{P}^{\{j\}}\right) \leq Q\left(\boldsymbol{\tau}^{\{j+1\}}, \boldsymbol{\alpha}^{\{j+1\}}, \mathbf{P}^{\{j+1\}}\right),
$$

which implies that the objective value of problem (10) is non-decreasing after each iteration in Algorithm 1 . Since the objective value of problem (10) is a finite positive value, the proposed Algorithm 1 is guaranteed to converge to the optimal objective value and solutions. This completes the convergence proof.

The complexity of Algorithm 1 is polynomial, since only three convex optimization problems need to be solved in each iteration. Hence, the proposed Algorithm 11 can be practically implemented with fast convergence for fullduplex $\mathrm{ABCNs}$ with a moderate number of BDs and $\mathrm{LU}(\mathrm{s})$.

\section{NUMERICAL RESULTS}

In this section, we provide simulation results to evaluate the performance of the proposed joint design. We consider an ABCN with $M=2$ BDs. Suppose that the FAP-toBD1 distance and FAP-to-BD2 distance are $2.5 \mathrm{~m}$ and $4 \mathrm{~m}$, respectively, the FAP (BD1, BD2)-to-LU distances are all $15 \mathrm{~m}$. We assume independent Rayleigh fading channels, i.e., the channel coefficient of each path is a circularly symmetric complex Gaussian prandom variable, and the power gains of multiple paths are exponentially distributed. For each channel link, its first-path channel power gain is assumed to be $10^{-3} d^{-2}$, where $d$ is the distance with unit of meter. Let the number of pathes $L_{\mathrm{f}}=L_{\mathrm{g}}=4, L_{\mathrm{h}}=8$, and $L_{\mathrm{v}}=6$. Other parameters are set as $N=64, N_{\mathrm{cp}}=$ $16, \bar{P}=1, \eta=0.5, \epsilon=10^{-4}$. Define the average receive SNR at the FAP as $\bar{\gamma}=\bar{P} \sum_{l=0}^{L_{\mathrm{f}}-1} \mathbb{E}\left[\left|g_{1, l} f_{1, l}\right|^{2}\right] / \sigma^{2}$. Let $E_{\min , 1}=E_{\min , 2}=E_{\min }$. For performance comparison, we consider a benchmark scheme in which the backscatter time portion and subcarrier power are equally allocated, i.e., $\tau_{m}=\frac{1}{M}, P_{m, k}=P_{\text {ave }}=\frac{1}{M N}$, and all BDs adopt a common power reflection coefficient that is optimized via CVX. The following results are obtainepd based on 100 random channel realizations.

Fig. 3 plots the max-min throughput of all BDs versus the LU's throughput requirement $D$ for different SNRs $\bar{\gamma}$ 's. We fix $P_{\text {peak }}=20 P_{\text {ave }}$ and $E_{\min }=10 \mu \mathrm{J}$. As expected, the max-min throughput decreases as $D$ increases, which reveals the throughput tradeoff between the BDs and the 


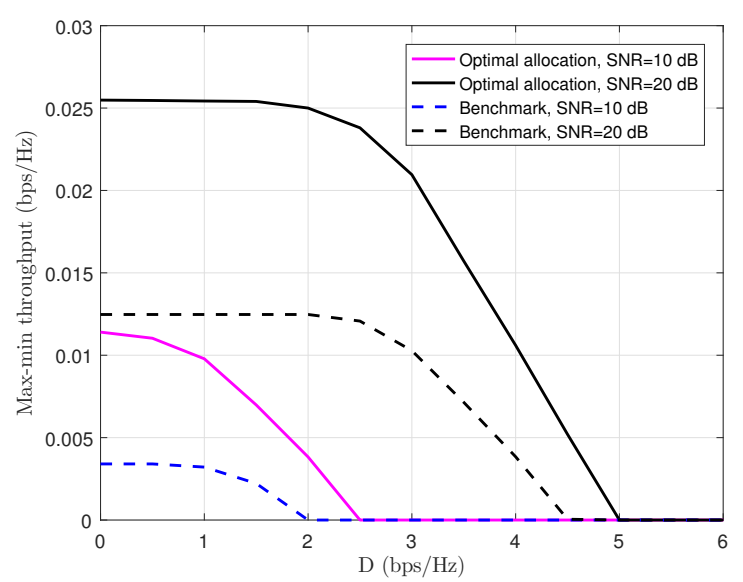

Fig. 3: Max-min throughput versus LU throughput requirement for different SNRs.

LU. We further observe that the max-min throughput performance is significantly enhanced by using the proposed joint design, compared to the benchmark scheme. Also, higher max-min throughput is achieved when the SNR at the FAP is higher.

Fig. 4 compares the max-min throughput under different BDs' energy requirements $E_{\min }$ 's and subcarrier peakpower values $P_{\text {peak }}$ 's, for both the proposed joint design and the benchmark scheme. We fix $D=1 \mathrm{bps} / \mathrm{Hz}$. In general, the max-min throughput increases as the SNR $\bar{\gamma}$ increases. We have three further observations. First and foremost, the proposed joint design achieves significant max-min throughput gains as compared to the benchmark scheme. Second, higher max-min throughput is achieved for lower harvested-energy requirement $E_{\text {min }}$ with given $P_{\text {peak }}$, which reveals the BDs' throughput-energy tradeoff. This observation can be specifically obtained from the three red solid curves for our proposed joint design and the three blue dotted curves for the benchmark scheme, given $P_{\text {peak }}=20 P_{\text {ave }}$. Third, higher max-min throughput is obtained for higher peak-power value $P_{\text {peak }}$ with given $E_{\text {min }}$, which is demonstrated in the red and black solid curves with triangle marker for our proposed joint design.

\section{CONCLUSION}

This paper has investigated a full-duplex AmBC network over ambient OFDM carriers. The minimum throughput among all BDs is maximized by jointly optimizing the BDs' backscatter time portions, the BDs' power reflection coefficients, and the FAP's subcarrier power allocation. By utilizing the block coordinated decent and successive convex optimization techniques, an efficient iterative algorithm is proposed, which is guaranteed to converge. Numerical results show that significant throughput gains are achieved as compared to the benchmark scheme with equal resource allocation, benefitting from multiple design dimensions of the proposed joint optimization. The interesting BDs'

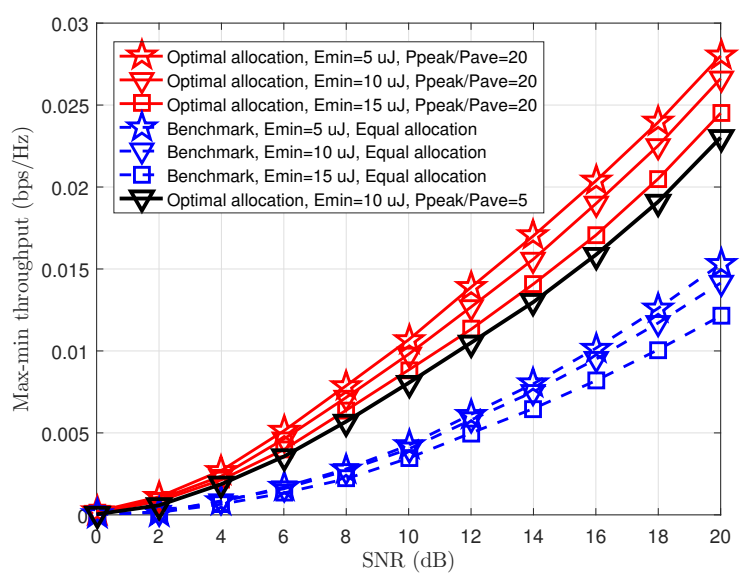

Fig. 4: Max-min throughput versus SNR for different harvested-energy requirements and peak power values.

throughput-energy tradeoff and the throughput tradeoff between the BDs and the LU are also revealed.

\section{REFERENCES}

[1] V. Liu, A. Parks, V. Talla, S. Gollakota, D. Wetherall, and J. R. Smith, "Ambient backscatter: wireless communication out of thin air," in Proc. of ACM SIGCOMM, Hong Kong, China, Jun. 2013, pp. 1-13.

[2] B. Kellogg, A. Parks, S. Gollakota, J. R. Smith, and D. Wetherall, "Wi-Fi backscatter: Internet connectivity for RF-powered devices," in Proc. of ACM SIGCOMM, Chicago, USA, Jun. 2014, pp. 1-12.

[3] G. Yang, Y.-C. Liang, R. Zhang, and Y. Pei, "Modulation in the air: Backscatter communication over ambient OFDM carrier," IEEE Trans. Commun., vol. 66, no. 3, pp. 1219-1233, Mar. 2018.

[4] G. Wang, F. Gao, R. Fan, and C. Tellambura, "Ambient backscatter communication systems: detection and performance analysis," IEEE Trans. Commun., vol. 64, no. 11, pp. 4836-4846, Nov. 2016.

[5] P. Zhang, M. Rostami, P. Hu, and D. Ganesan, "Enabling practical backscatter communication for on-body sensors," in Proc. of ACM SIGCOMM, Florianopolis, Brazil, Aug. 2016, pp. 370-383.

[6] A. Wang, V. Iyer, V. Talla, J. R. Smith, and S. Gollakota, "FM backscatter: Enabling connected cities and smart fabrics," in Proc. of USENIX Symposium on Networked Systems Design and Implementation (NSDI), Boston, MA, USA, Mar. 2017, pp. 1-16.

[7] G. Yang, Q. Zhang, and Y.-C. Liang, "Cooperative ambient backscatter communications for green internet-of-things," IEEE Internet-of-Things Journal, vol. 5, no. 2, pp. 1116-1130, Apr. 2018.

[8] R. Long, G. Yang, Y. Pei, and R. Zhang, "Transmit beamforming for cooperative ambient backscatter communication systems," in Proc. of IEEE GLOBECOM, Singapore, December 2017, pp. 1-6.

[9] D. Bharadia, K. Joshi, M. Kotaru, and S. Katti, "BackFi: High throughput WiFi backscatter," in Proc. of ACM SIGCOMM, London, UK, Aug. 2015, pp. 283-296.

[10] X. Kang, Y.-C. Liang, and J. Yang, "Riding on the primary: A new spectrum sharing paradigm for wireless-powered IoT devices," in Proc. of IEEE Conf. Commun. (ICC), Paris, France, May 2017, pp. $1-6$.

[11] D. T. Hoang, D. Niyato, P. Wang, D. I. Kim, and Z. Han, "Ambient backscatter: A new approach to improve network performance for rf-powered cognitive radio networks," IEEE Trans. Commun., vol. 65 , no. 9, pp. $3659-3674$, Sep. 2017.

[12] X. Zhou, R. Zhang, and C. K. Ho, "Wireless information and power transfer in multiuser OFDM systems," IEEE Wireless Commun., vol. 13, no. 4, pp. 2282-2294, Apr. 2014.

[13] M. Hong, M. Razaviyayn, Z.-Q. Luo, and J.-S. Pang, "A unified algorithmic framework for block-structured optimization involving big data: With applications in machine learning and signal processing," IEEE Signal Process. Mag., vol. 33, no. 1, pp. 57-77, Jan. 2016.

[14] A. Beck, A. Ben-Tal, and L. Tetruashvili, "A sequential parametric convex approximation method with applications to nonconvex truss topology design problems," Journal of Global Optimization, vol. 47, no. 1, pp. 29-51, May 2010.

[15] M. Grant and S. Boyd, "CVX: Matlab software for disciplined convex programming," [Online]. Available: http://cvxr.com/cvx 